\title{
Noncommutativity in space-time
}

\section{extended by Liouville field}

\author{
Bojan Nikolić and Branislav Sazdović \\ Institute of Physics, 11001 Belgrade, P.O.Box 57, Serbia \\ bnikolic@ipb.ac.rs, sazdovic@ipb.ac.rs
}

\begin{abstract}
The world-sheet quantum conformal invariance can be realized in the presence of the conformal factor $F$ by inclusion of the Liouville term. In the background with linear dilaton field, $\Phi(x)=\Phi_{0}+a_{\mu} x^{\mu}$, the field $F$ becomes a new noncommutative variable. Therefore, it is natural to extend space-time with a new coordinate, $F$, in order to unify expressions for noncommutativity parameter $\Theta^{i j}$ of the $D p$-brane space-time coordinates $x^{i}$, with the part $\Theta^{i}$ connecting noncommutativity between coordinates $x^{i}$ and $F$. In this way we solve the problems of $D p$-brane noncommutativity in a more elegant way. The technical advantage is in the fact that in the extended space-time the action with dilaton field can be rewritten in dilaton free form.

We use canonical method and extend its application to the derivation of boundary conditions. From requirement that Hamiltonian, as the time translation generator, has well-defined derivatives in the coordinates and momenta, we obtain boundary conditions directly in the canonical form.
\end{abstract}

e-print archive: http://lanl.arXiv.org/abs/hep-th//0711.4463 


\section{Introduction}

In $[1,2]$ we explained new possibility in order to realize world-sheet quantum conformal invariance. Instead of standard requirement for vanishing $\beta$-functions corresponding to all background fields (metric, antisymmetric tensor, and dilaton field), $\beta_{\mu \nu}^{G}=\beta_{\mu \nu}^{B}=\beta^{\Phi}=0$, we used the fact established in [3] that condition $\beta_{\mu \nu}^{G}=0$ implies that the third one is constant, $\beta^{\Phi}=c$. This constant contribution to the conformal anomaly has been cancelled by adding Lioville term. Then the theory depends on arbitrary central charge $c$, and the conformal invariance is realized in the presence of the conformal factor of the world-sheet metric, $F$. The Liouville action brings dynamics to the field $F$. So, in open string theory we should choose its boundary condition and investigate its contribution to standard results of noncommutativity without dilaton field [4-6]. It is shown in $[1,2,7]$ that in the presence of linear dilaton field, $\Phi(x)=\Phi_{0}+a_{i} x^{i}$ (which initially has been investigated in [8]), the field $F$ is a new noncommutative dynamical variable, while $D p$-brane coordinate $x_{\mathrm{c}}=a_{i} x^{i}$ is a commutative one.

In order to unify the expressions for noncommutativity parameters of $[1,2,7]$ we are going to treat the conformal part of the world-sheet metric $F$ as an additional coordinate of some extended space-time with the coordinates $y^{M}=\left(x^{\mu}, F\right)$ and the metric $G_{M N}$ depending on ordinary metric $G_{\mu \nu}$, dilaton gradient $a_{\mu}=\partial_{\mu} \Phi$ and central charge $c$. We will investigate the geometry and noncommutativity features of extended $D p$-brane, embedded in the extended space-time, and parameterized by the extended coordinates $y^{A}=\left(x^{i}, F\right)$, consisting of $D p$-brane space-time coordinates $x^{i}$ and field $F$, with the corresponding extended metric tensor $G_{A B}$. In the extended formulation the starting action obtains the dilaton free form, which simplifies all calculations. Such approach makes the previous results more transparent and offers their geometrical interpretation.

We use notation and terminology of [2] distinguishing two descriptions of the same open string theory. In terms of variable $y^{A}$ and background field $G_{A B}$, the theory is described by equations of motion and boundary conditions. The effective theory, defined on solution of boundary conditions, is described only by effective equations of motion. This is the string theory on orbifolds expressed in terms of effective variables $q^{A}$ symmetric under transformation $\sigma \rightarrow-\sigma$ and effective background field $G_{A B}^{\text {eff }}$. We extend terminology of [6] to the extended theory referring to metric $G_{A B}$ as closed string metric and to $G_{A B}^{\mathrm{eff}}$ as open string metric.

As a difference of standard $D p$-brane metric $G_{i j}$, which is always regular, the extended metrics can be singular for some particular relations between 
background fields. So, we investigate three cases: (1) both closed and open extended metrics are regular, (2) extended closed string metric $G_{A B}$ is singular, and (3) extended open string metric $G_{A B}^{\mathrm{eff}}$ is singular.

The singularities of the extended metrics produce the first class constraints, but with different origins. In the case (2) singular $G_{A B}$ produce a standard constraint of the Dirac type. In the case (3) singularity of $G_{A B}^{\mathrm{eff}}$ turns some second class constraints, obtained from the boundary conditions, into the first class ones. In fact, Poisson brackets of these constraints close on the extended effective metric $G_{A B}^{\mathrm{eff}}$. The singular directions of this metric project complete set of constraints to the first class ones.

The first class constraints generate local gauge symmetries. After gauge fixing the first class constraints and gauge conditions can be treated as second class constraints. Solving these constraints together with the remaining second class constraints, we obtain effective theory.

There is also an important methodological improvement. In Section 3.2 we obtain the boundary conditions purely canonically, from the requirement that Hamiltonian is differentiable in its canonical variables. This is more natural approach because we intend to treat these conditions as canonical constraints. The equations of motion for the momenta turn canonical boundary conditions to the standard Lagrangian ones.

The last part of the article contains concluding remarks and two appendices. Appendix $\mathrm{A}$ is devoted to the closed and open string metrics of the extended space-time, and the corresponding zero central charge limit $(c=0)$. In Appendix B we introduce the projectors which help us to express the results clearly.

\section{Extended $D p$-brane in extended space-time}

The action that describes dynamics of the open string in the presence of the space-time metric $G_{\mu \nu}(x)$, Kalb-Ramond antisymmetric field $B_{\mu \nu}(x)$, and dilaton scalar field $\Phi(x)$ is of the form [9]

$$
\begin{aligned}
S_{(G+B+\Phi)}= & \kappa \int_{\Sigma} d^{2} \xi \sqrt{-g}\left\{\left[\frac{1}{2} g^{\alpha \beta} G_{\mu \nu}(x)+\frac{\varepsilon^{\alpha \beta}}{\sqrt{-g}} B_{\mu \nu}(x)\right]\right. \\
& \left.\times \partial_{\alpha} x^{\mu} \partial_{\beta} x^{\nu}+\Phi(x) R^{(2)}\right\} .
\end{aligned}
$$


We integrate in the action over the world-sheet surface $\Sigma$ parameterized by $\xi^{\alpha}=(\tau, \sigma)[(\alpha=0,1), \sigma \in(0, \pi)]$, while the $D$-dimensional space-time is spanned by the coordinates $x^{\mu}(\mu=0,1,2, \ldots, D-1)$. We denote intrinsic world-sheet metric with $g_{\alpha \beta}$, and the corresponding scalar curvature with $R^{(2)}$.

Three $\beta$-functions characterize the conformal anomaly of the sigma model (2.1)

$$
\begin{gathered}
\beta_{\mu \nu}^{G} \equiv R_{\mu \nu}-\frac{1}{4} B_{\mu \rho \sigma} B_{\nu}{ }^{\rho \sigma}+2 D_{\mu} a_{\nu}, \\
\beta_{\mu \nu}^{B} \equiv D_{\rho} B^{\rho}{ }_{\mu \nu}-2 a_{\rho} B^{\rho}{ }_{\mu \nu}, \\
\beta^{\Phi} \equiv 2 \pi \kappa \frac{D-26}{6}-R-\frac{1}{24} B_{\mu \rho \sigma} B^{\mu \rho \sigma}-D_{\mu} a^{\mu}+4 a^{2},
\end{gathered}
$$

where $R_{\mu \nu}$ and $D_{\mu}$ are Ricci tensor and covariant derivative with respect to the space-time metric $G_{\mu \nu}$, while

$$
B_{\mu \nu \rho}=\partial_{\mu} B_{\nu \rho}+\partial_{\nu} B_{\rho \mu}+\partial_{\rho} B_{\mu \nu}, \quad a_{\mu}=\partial_{\mu} \Phi .
$$

It is shown in [3], that vanishing of $\beta_{\mu \nu}^{G}$ and $\beta_{\mu \nu}^{B}$ gives constant value of the third $\beta$-function, $\beta^{\Phi}=c$, and the non-linear sigma model (2.1) becomes conformal field theory. Therefore, Virasoro algebra with central charge $c$ emerges.

From this point our approach differs from the previous one [1]. We retain two conditions, $\beta_{\mu \nu}^{G}=0$ and $\beta_{\mu \nu}^{B}=0$, but in order to cancel the remaining conformal anomaly we add the corresponding Wess-Zumino term to the action (2.1). In this concrete case the role of Wess-Zumino term takes the Liouville action

$$
S_{\mathrm{L}}=-\frac{\beta^{\Phi}}{2(4 \pi)^{2} \kappa} \int_{\Sigma} d^{2} \xi \sqrt{-g} R^{(2)} \frac{1}{\Delta} R^{(2)}, \quad \Delta=g^{\alpha \beta} \nabla_{\alpha} \partial_{\beta},
$$

where with $\nabla_{\alpha}$ we denote the covariant derivative with respect to the intrinsic metric $g_{\alpha \beta}$. Note, in this approach we establish quantum conformal invariance even in the presence of the field $F$. The complete action, in the conformal gauge $g_{\alpha \beta}=\mathrm{e}^{2 F} \eta_{\alpha \beta}$, takes the form

$$
\begin{aligned}
S= & \kappa \int_{\Sigma} d^{2} \xi\left[\left(\frac{1}{2} \eta^{\alpha \beta} G_{\mu \nu}+\epsilon^{\alpha \beta} B_{\mu \nu}\right) \partial_{\alpha} x^{\mu} \partial_{\beta} x^{\nu}\right. \\
& \left.+2 \eta^{\alpha \beta} a_{\mu} \partial_{\alpha} x^{\mu} \partial_{\beta} F+\frac{2}{\alpha} \eta^{\alpha \beta} \partial_{\alpha} F \partial_{\beta} F\right]
\end{aligned}
$$


where we defined the parameter $\alpha$ as

$$
\frac{1}{\alpha}=\frac{\beta^{\Phi}}{(4 \pi \kappa)^{2}} .
$$

The space-time with coordinates $x^{\mu}(\xi)$ is decomposed in $D p$-brane part spanned by coordinates $x^{i}(\xi)(i=0,1, \ldots, p)$, and the orthogonal ones, $x^{a}(\xi)$ $(a=p+1, p+2, \ldots, D-1)$, in such a way that $G_{\mu \nu}=0,(\mu=i, \nu=a)$. Also we choose the case where the fields $B_{\mu \nu}$ and $a_{\mu}$ live only on the $D p$ brane: $B_{\mu \nu} \rightarrow B_{i j}, a_{\mu} \rightarrow a_{i}$.

In order to simplify the calculations and to offer geometrical unification of noncommutativity parameters, it is useful to introduce an extended spacetime defined by the coordinates

$$
y^{M}=\left(x^{\mu}, F\right)=\left(y^{A}, x^{a}\right),
$$

where an extended $D p$-brane is parameterized by

$$
y^{A}=\left(x^{i}, F\right), \quad A \in\{0,1, \ldots, p+1\} .
$$

The part of the action describing the string oscillation in $x^{a}$ directions decouples. We will analyze the rest part described by action

$$
S=\kappa \int_{\Sigma} d^{2} \xi\left[\frac{1}{2} \eta^{\alpha \beta} G_{A B}+\epsilon^{\alpha \beta} B_{A B}\right] \partial_{\alpha} y^{A} \partial_{\beta} y^{B},
$$

where the corresponding background fields $G_{A B}$ and $B_{A B}$ are defined in equation (A.1). Let us stress that the action in the presence of dilaton field in extended space-time has the form of dilaton free action.

The action (2.11) can be rewritten in the form

$$
S=\kappa \int_{\Sigma} d^{2} \xi\left[\frac{1}{2} \eta^{\alpha \beta \star} G_{A B}+\epsilon^{\alpha \beta} B_{A B}\right] \partial_{\alpha}{ }^{\star} y^{A} \partial_{\beta}{ }^{\star} y^{B},
$$

where the extended metric ${ }^{\star} G_{A B}$ (A.5) is diagonal, and ${ }^{\star} y^{A}=\left(x^{i},{ }^{\star} F\right)$ is defined in equation (A.4). Because these two forms of metric are connected by similarity transformation (A.3), further we will use the same notation for both of them omitting the mark $(\star)$. The first form is useful for zero central charge limit $c=\beta^{\Phi}=0(\alpha \rightarrow \infty)$, where Liouville field disappears. The advantages of the second form are diagonalization of the extended metric and manifest separation of one variable, ${ }^{\star} F$, which simplify the comparison with results of [2]. 
All nontrivial features of the model defined in equations (2.11) and (2.12) follow from the fact that the extended metrics $\left(G_{A B}\right.$ and the corresponding effective one $G_{A B}^{\text {eff }}$ ) are singular for some specific choices of the background fields. It is easy to check that for $\mathcal{A} \equiv \frac{1}{\alpha}-a^{2}=0$ and $\tilde{\mathcal{A}} \equiv \frac{1}{\alpha}-\tilde{a}^{2}=0$ we have $\operatorname{det} G_{A B}=0$ and $\operatorname{det} G_{A B}^{\mathrm{eff}}=0$, respectively.

\section{Canonical analysis of open string theory}

Because boundary conditions will be treated as canonical constraints, we will derive them in terms of coordinate and momenta using canonical method. In this section we assume that extended metric $G_{A B}$ is regular, while particular cases of singular extended metrics are discussed in Section 4.

\subsection{Canonical Hamiltonian in terms of currents}

The momenta canonically conjugated to the fields $y^{A}$ are

$$
\pi_{A}=\kappa\left(G_{A B} \dot{y}^{B}-2 B_{A B} y^{\prime B}\right) .
$$

Using the definition of the canonical Hamiltonian $\mathcal{H}_{\mathrm{c}}=\pi_{A} \dot{y}^{A}-\mathcal{L}$, we obtain

$$
H_{\mathrm{c}}=\int d \sigma \mathcal{H}_{\mathrm{c}}, \quad \mathcal{H}_{\mathrm{c}}=T_{-}-T_{+}, \quad T_{ \pm}=\mp \frac{1}{4 \kappa}\left(G^{-1}\right)^{A B} j_{ \pm A} j_{ \pm B}
$$

where the expression for inverse metric $\left(G^{-1}\right)^{A B}$ is given in equation (A.6), and the current is defined as

$$
j_{ \pm A}=\pi_{A}+2 \kappa \Pi_{ \pm A B} y^{\prime B} \quad\left(\Pi_{ \pm A B}=B_{A B} \pm \frac{1}{2} \cdot G_{A B}\right) .
$$

From the basic Poisson bracket algebra

$$
\left\{y^{A}(\tau, \sigma), \pi_{B}(\tau, \bar{\sigma})\right\}=\delta^{A}{ }_{B} \delta(\sigma-\bar{\sigma}),
$$

the current algebra directly follows

$$
\left\{j_{ \pm A}, j_{ \pm B}\right\}= \pm 2 \kappa G_{A B} \delta^{\prime}
$$

while all opposite chirality currents commute and for simplicity we define $\delta^{\prime} \equiv \partial_{\sigma} \delta(\sigma-\bar{\sigma})$. Consequently, the Poisson bracket between canonical 
Hamiltonian and the current $j_{ \pm A}$ is proportional to its sigma derivative

$$
\left\{H_{\mathrm{c}}, j_{ \pm A}\right\}=\mp j_{ \pm A}^{\prime} .
$$

This canonical analysis is formally equivalent to the analysis without dilaton field. But, because we work in extended space-time, it contains dilaton. The components of the currents and energy-momentum tensor

$$
\begin{aligned}
j_{ \pm}^{A} & =G^{A B} j_{ \pm B}=\left(\begin{array}{c}
J_{ \pm}^{i} \\
\frac{1}{2} i_{ \pm}^{F}
\end{array}\right), \quad J_{ \pm}^{i}=\left(G^{i j}+\frac{a^{i} a^{j}}{\mathcal{A}}\right) j_{ \pm j}-\frac{a^{i}}{2 \mathcal{A}} i_{ \pm}^{\Phi} \\
i_{ \pm}^{F} & =-\frac{1}{\mathcal{A}}\left(a^{i} j_{ \pm i}-\frac{1}{2} i_{ \pm}^{\Phi}\right) \\
T_{ \pm} & =\mp \frac{1}{4 \kappa}\left[G_{i j} J_{ \pm}^{i} J_{ \pm}^{j}+2\left(a_{i} J_{ \pm}^{i}\right) i_{ \pm}^{F}+\frac{1}{\alpha} i_{ \pm}^{F} i_{ \pm}^{F}\right]
\end{aligned}
$$

where

$$
j_{ \pm A}=\left(\begin{array}{c}
j_{ \pm i} \\
i_{ \pm}^{\Phi}
\end{array}\right)=\left(\begin{array}{c}
\pi_{i}+2 \kappa \Pi_{ \pm i j} x^{\prime j} \pm 2 \kappa a_{i} F^{\prime} \\
\pi \pm 2 \kappa a_{i} x^{\prime i} \pm \frac{4 \kappa}{\alpha} F^{\prime}
\end{array}\right)
$$

for $c=0(\alpha \rightarrow \infty)$, are in full agreement with the corresponding ones of [10].

\subsection{Hamiltonian derivation of the boundary conditions}

In $[5,7]$ the open string boundary conditions have been introduced in Lagrangian formalism and rewritten in terms of canonical variables. Because we intend to treat the open string boundary conditions as canonical constraints, we will derive them directly in Hamiltonian form.

The Hamiltonian is a generator of the time translation, so it must be differentiable in coordinates and momenta. Varying Hamiltonian (3.2), we obtain

$$
\delta H_{\mathrm{c}}=\delta H_{\mathrm{c}}^{(R)}-\left.\gamma_{A}^{(0)} \delta y^{A}\right|_{0} ^{\pi},
$$

where index $R$ denotes the regular term of the form

$$
\delta H_{\mathrm{c}}^{(R)}=\int d \sigma\left(A_{A} \delta y^{A}+B^{A} \delta \pi_{A}\right)
$$


and

$$
\gamma_{A}^{(0)}=\left(\Pi_{-} G^{-1}\right)_{A}^{B} j_{+B}+\left(\Pi_{+} G^{-1}\right)_{A}^{B} j_{-B}
$$

The Hamiltonian is properly defined canonical variable, when the boundary term $\left.\gamma_{A}^{(0)} \delta y^{A}\right|_{0} ^{\pi}$ in equation (3.10) vanishes. That is automatically fulfilled for closed strings, because they do not have endpoints. Assuming that the variations $\delta y^{A}$ are arbitrary at the open string endpoints, we obtain the Neumann boundary conditions in the canonical form, $\left.\gamma_{A}^{(0)}\right|_{0} ^{\pi}=0$. On the other hand, if we suppose that the string endpoints are fixed, $\left.\delta y^{A}\right|_{0} ^{\pi}=0$, the boundary conditions are known as Dirichlet boundary conditions. We choose the Neumann boundary conditions for variables $y^{A}$. Note that beside the Neumann boundary conditions on coordinates $x^{i}$ it also includes the Neumann boundary condition on Liouville field $F$.

After imposing the expressions for momenta obtained on their equations of motion, the boundary conditions reduce to the Lagrangian ones of $[5,7]$

$$
\gamma_{A}^{(0)}=\kappa\left(-G_{A B} y^{\prime B}+2 B_{A B} \dot{y}^{B}\right)
$$

Checking the consistency of the constraints, with the help of relation (3.6), we obtain an infinite set of constraints. Using Taylor expansion, we rewrite all the constraints at $\sigma=0$ in a more compact $\sigma$-dependent form

$$
\Gamma_{A}(\sigma)=\sum_{n \geq 0} \frac{\sigma^{n}}{n !} \gamma_{A}^{(n)}(\sigma=0)=\left(\Pi_{+} G^{-1}\right)_{A}^{B} j_{-B}(\sigma)+\left(\Pi_{-} G^{-1}\right)_{A}{ }^{B} j_{+B}(-\sigma)
$$

where

$$
\gamma_{A}^{(n)} \equiv\left\{H_{\mathrm{c}}, \gamma_{A}^{(n-1)}\right\}=\left(\Pi_{+} G^{-1}\right)_{A}^{B} \partial_{\sigma}^{n} j_{-B}+(-1)^{n}\left(\Pi_{-} G^{-1}\right)_{A}{ }^{B} \partial_{\sigma}^{n} j_{+B} .
$$

In the same way, we obtain similar expressions from the constraints at $\sigma=$ $\pi$. From the fact that the differences of the corresponding constraints at $\sigma=0$ and $\pi$ are also constraints, we conclude that all positive chirality currents and, consequently, all variables are $2 \pi$ periodic functions. Because of this periodicity the constraints at $\sigma=\pi$ can be discarded (for more details see [7]). 
We complete the consistency procedure finding the Poisson bracket

$$
\left\{H_{\mathrm{c}}, \Gamma_{A}\right\}=\Gamma_{A}^{\prime},
$$

which means that there are no more constraints in the theory.

\section{First class constraints and gauge symmetries}

In order to finish canonical analysis we have to classify the constraints. The nature of the constraints depends on the (non)singularity of the extended metrics, $G_{A B}$ and $G_{A B}^{\mathrm{eff}}$. It turns out that, for some particular choices of the background fields, they have vanishing determinants, which produce the first class constraints. According to the Dirac theory for constrained systems, the first class constraints generate gauge symmetries in the theory, which existence enables us to fix nonphysical degrees of freedom.

\subsection{Case of regular metrics $(\mathcal{A} \neq 0, \tilde{\mathcal{A}} \neq 0)$}

For $\mathcal{A} \neq 0$ we have

$$
\operatorname{det} G_{A B}=4 \mathcal{A} \operatorname{det} G_{i j} \neq 0,
$$

so that we are able to solve all velocities in terms of momenta from equation (3.1). In that case there are no constraints of Dirac type.

The algebra of the constraints $\Gamma_{A}$ originating from boundary conditions has a simple matrix form

$$
\left\{\Gamma_{A}(\sigma), \Gamma_{B}(\bar{\sigma})\right\}=-\kappa G_{A B}^{\mathrm{eff}} \delta^{\prime},
$$

where $G_{A B}^{\mathrm{eff}}$ is defined in equations (A.9) and (A.10). The determinant of $G_{A B}^{\text {eff }}$

$$
\operatorname{det} G_{A B}^{\mathrm{eff}}=4 \frac{\tilde{\mathcal{A}}^{2}}{\mathcal{A}} \operatorname{det} G_{i j}^{\mathrm{eff}}
$$

is regular for $\tilde{\mathcal{A}} \neq 0$ and $\mathcal{A} \neq 0$, and all constraints are of the second class. We use the assumption that the standard metrics are regular, $\operatorname{det} G_{i j} \neq 0$ and $\operatorname{det} G_{i j}^{\text {eff }} \neq 0$. 


\subsection{Singularity of the metric $G_{A B}(\mathcal{A}=0)$}

For $\mathcal{A}=0$ the determinant of the metric $G_{A B}$ (4.1) is equal to zero. This means that extended metric $G_{A B}$ have one singular direction $n^{A}$ defined in equation (B.4). From (3.1) it is clear, that we are not able to solve all velocities in terms of momenta, and consequently there must be a primary constraint. For $\mathcal{A}=0$, the current

$$
j \equiv n^{A} j_{ \pm A}=n^{A} \pi_{A}+2 \kappa n^{A} B_{A B} y^{\prime B}
$$

does not depend on velocities and we conclude that it is a constraint. From the algebra of currents (3.5) we obtain that $j$ commutes with all $j_{ \pm A}$, so it is of the first class.

The canonical Hamiltonian is of the form

$$
H_{\mathrm{c}}=\int d \sigma \mathcal{H}_{\mathrm{c}}, \quad \mathcal{H}_{\mathrm{c}}=T_{-}-T_{+}, \quad T_{ \pm}=\mp \frac{1}{4 \kappa}\left(g^{-1}\right)^{A B} j_{ \pm A} j_{ \pm B},
$$

where $\left(g^{-1}\right)^{A B}$ defined in (B.9) is the inverse of the induced metric in the subspace orthogonal to the vector $n^{A}$. In order to examine the consistency of the constraint $j$ we introduce the total Hamiltonian

$$
H_{\mathrm{T}}=H_{\mathrm{c}}+\int d \sigma \lambda(\sigma) j(\sigma)
$$

where $\lambda$ is a Lagrange multiplier. From the equation

$$
\left\{H_{\mathrm{T}}, j\right\} \approx 0,
$$

we conclude that there are no more constraints in the theory and the multiplier $\lambda$ remains undetermined. This confirms that $j$ is a constraint of the first class.

The first class constraints generates the local gauge symmetry transformations of an arbitrary variable $X$

$$
\delta X=\{X, G\}, \quad G=\int d \sigma \eta(\sigma) j(\sigma) .
$$

If we apply this to the coordinates $y^{A}$ and canonically conjugated momenta $\pi_{A}$, we get

$$
\delta y^{A}=n^{A} \eta, \quad \delta \pi_{A}=2 \kappa n^{B} B_{B A} \eta^{\prime} .
$$


From the expression

$$
\delta y \equiv \delta\left(\frac{n_{A} y^{A}}{n^{2}}\right)=\eta, \quad n^{2}=G_{A B} n^{A} n^{B},
$$

we conclude that $y=0$ is a good gauge condition. Note that

$$
n_{A} y^{A}=\alpha \mathcal{A} a_{i} x^{i}, \quad n^{2}=\alpha \mathcal{A} a^{2},
$$

are equal to zero for $\mathcal{A}=0$, but their fraction does not depend on $\mathcal{A}$ and, consequently, it is finite.

Using the constraint equation $j=0$ and gauge fixing $y=0$ the boundary conditions take the form

$$
\Gamma_{A}(\sigma) \rightarrow \check{\Gamma}_{A}=\left(\Pi_{+} g^{-1}\right)_{A}^{B} j_{-B}(\sigma)+\left(\Pi_{-} g^{-1}\right)_{A}{ }^{B} j_{+B}(-\sigma),
$$

with the algebra

$$
\left\{\check{\Gamma}_{A}(\sigma), \check{\Gamma}_{B}(\bar{\sigma})\right\}=-\kappa \check{G}_{A B}^{\mathrm{eff}} \delta^{\prime}
$$

The extended effective metric has the form

$$
\begin{aligned}
\check{G}_{A B}^{\mathrm{eff}} & =\left.\left(-2\left[\left(\Pi_{+} g^{-1} \Pi_{-}\right)+\left(\Pi_{-} g^{-1} \Pi_{+}\right)\right]_{A B}\right)\right|_{\mathcal{A}=0} \\
& =\left.\left(g_{A B}-4\left(B g^{-1} B\right)_{A B}\right)\right|_{\mathcal{A}=0}
\end{aligned}
$$

with the concrete expression given in equation (B.14). For $\tilde{a}^{2} \neq 0$ and $\tilde{\mathcal{A}} \neq 0$ with the help of the relation

$$
\operatorname{det} \check{G}_{A B}^{\mathrm{eff}}=4 \alpha \tilde{a}^{2} \tilde{\mathcal{A}} \operatorname{det} G_{i j}^{\mathrm{eff}},
$$

we conclude that all constraints originating from boundary conditions and remaining after gauge fixing are of the second class.

\subsection{Singularity of the metric $G_{A B}^{\mathrm{eff}}(\tilde{\mathcal{A}}=0)$}

From expression (4.3) we conclude that, for $\tilde{\mathcal{A}}=0$ and $\mathcal{A} \neq 0$, $\operatorname{det} G_{A B}^{\text {eff }}$ has two zeros at $\tilde{\mathcal{A}}=0$, which means that metric $G_{A B}^{\text {eff }}$ has two singular directions $\tilde{n}_{1}^{A}$ and $\tilde{n}_{2}^{A}$ introduced in equations (B.10) and (B.11). According 
to the algebra of constraints $(4.2)$, for $\tilde{\mathcal{A}}=0$ and $\mathcal{A} \neq 0$, the constraints

$$
\Gamma_{1}=\tilde{n}_{1}^{A} \Gamma_{A}, \quad \Gamma_{2}=\tilde{n}_{2}^{A} \Gamma_{A},
$$

are of the first class, while the rest ones

$$
\left(\Gamma_{\mathrm{T}}\right)_{A}=\left(\hat{P}_{\mathrm{T}}\right)_{A}{ }^{B} \Gamma_{B}
$$

are of the second class. The projector $\left(\hat{P}_{\mathrm{T}}\right)_{A}{ }^{B}$, introduced in equation (B.13), projects on the subspace orthogonal to directions $\tilde{n}_{1}^{A}$ and $\tilde{n}_{2}^{A}$.

The generator corresponding to the first class constraints $\Gamma_{1}$ and $\Gamma_{2}$ is

$$
G=\int d \sigma\left[\eta_{1}(\sigma) \Gamma_{1}(\sigma)+\eta_{2}(\sigma) \Gamma_{2}(\sigma)\right],
$$

with the same form of gauge transformation as in equation (4.8). Using the identities

$$
\tilde{n}_{1}^{A}=2\left(\tilde{n}_{2} B G^{-1}\right)^{A}, \quad \tilde{n}_{2}^{A}=2\left(\tilde{n}_{1} B G^{-1}\right)^{A},
$$

we obtain that the constraints do not depend on the coordinate $y^{A}$

$$
\begin{aligned}
& \Gamma_{1}=\frac{1}{2}\left(\tilde{n}_{1}^{A}+\tilde{n}_{2}^{A}\right) \pi_{A}(\sigma)+\frac{1}{2}\left(\tilde{n}_{2}^{A}-\tilde{n}_{1}^{A}\right) \pi_{A}(-\sigma), \\
& \Gamma_{2}=\frac{1}{2}\left(\tilde{n}_{1}^{A}+\tilde{n}_{2}^{A}\right) \pi_{A}(\sigma)+\frac{1}{2}\left(\tilde{n}_{1}^{A}-\tilde{n}_{2}^{A}\right) \pi_{A}(-\sigma) .
\end{aligned}
$$

Consequently, the gauge transformations of the momenta $\pi_{A}$ are trivial, and we obtain

$$
\begin{aligned}
\delta y^{A}= & \frac{1}{2}\left(\tilde{n}_{1}^{A}+\tilde{n}_{2}^{A}\right)\left[\eta_{1}(\sigma)+\eta_{2}(\sigma)\right] \\
& +\frac{1}{2}\left(\tilde{n}_{2}^{A}-\tilde{n}_{1}^{A}\right)\left[\eta_{1}(-\sigma)-\eta_{2}(-\sigma)\right], \quad \delta \pi_{A}=0
\end{aligned}
$$

The particular gauge transformations

$$
\begin{gathered}
\delta y_{1}=\frac{1}{2}\left[\eta_{2}(\sigma)+\eta_{2}(-\sigma)\right]+\frac{1}{2}\left[\eta_{1}(\sigma)-\eta_{1}(-\sigma)\right] \\
\delta y_{2}=\frac{1}{2}\left[\eta_{1}(\sigma)+\eta_{1}(-\sigma)\right]+\frac{1}{2}\left[\eta_{2}(\sigma)-\eta_{2}(-\sigma)\right], \\
y_{a} \equiv \frac{\tilde{n}_{a A} y^{A}}{\tilde{n}_{a}^{2}}, \quad \tilde{n}_{a}^{2}=G_{A B}^{\mathrm{eff}} \tilde{n}_{a}^{A} \tilde{n}_{a}^{B}(a=1,2),
\end{gathered}
$$

enable us to choose good gauge conditions

$$
y_{1}=0, \quad y_{2}=0
$$

As well as in equation (4.11) $\tilde{n}_{a A} y^{A}$ and $\tilde{n}_{a}^{2}$ vanish for $\tilde{\mathcal{A}}=0$, but the variable $y_{a}$ is well defined. 


\section{$5 \quad D p$-brane features}

Using the solution of the second class constraints, we obtain that Poisson brackets of some coordinates are non-zero. The effective theory, defined on these constraints, describes string symmetric under $\sigma$-parity, which propagates in new, so-called effective background. In the zero central charge limit, $c=0$, we get the full agreement with the corresponding results of [1].

\subsection{Solution of constraint equations}

In the case of regular metrics we solve second class constraints originating from boundary conditions (3.14), $\Gamma_{A}=0$. In the cases of singular metrics, the first class constraints and local gauge symmetry appear. After gauge fixing we can treat the first class constraints and gauge conditions as second class constraints. For $\mathcal{A}=0$ we solve gauge condition $y=0$, first class constraint $j=0$, and, the second class constraints $\check{\Gamma}_{A}=0$ (4.12). In the case $\tilde{\mathcal{A}}=0$, solution of the the first class constraints, $\Gamma_{1}=0$ and $\Gamma_{2}=0$, and the second class ones, $\left(\Gamma_{\mathrm{T}}\right)_{A}=0$, is equivalent to the solution of all constraints, $\Gamma_{A}=0$. Then the complete set of equation consists of $\Gamma_{A}=0$ and the gauge conditions, $y_{1}=0$ and $y_{2}=0,(4.24)$.

In terms of the $\sigma$-symmetric and antisymmetric parts of coordinates and momenta

$$
\begin{array}{ll}
q^{A}(\sigma)=\frac{1}{2}\left[y^{A}(\sigma)+y^{A}(-\sigma)\right], & \bar{q}^{A}(\sigma)=\frac{1}{2}\left[y^{A}(\sigma)-y^{A}(-\sigma)\right], \\
p_{A}(\sigma)=\frac{1}{2}\left[\pi_{A}(\sigma)+\pi_{A}(-\sigma)\right], & \bar{p}_{A}(\sigma)=\frac{1}{2}\left[\pi_{A}(\sigma)-\pi_{A}(-\sigma)\right],
\end{array}
$$

the constraints $\Gamma_{A}(\sigma)$ have the form

$$
\Gamma_{A}=2\left(B G^{-1}\right)_{A}^{B} p_{B}-\kappa G_{A B}^{\mathrm{eff}} \bar{q}^{B}+\bar{p}_{A} .
$$

Solving the corresponding set of equations, we obtain that the solution in all three considered cases has the same form

$$
y_{\mathrm{Dp}}^{A}(\sigma)=Q^{A}(\sigma)-2 \Theta^{A B} \int_{0}^{\sigma} d \sigma_{1} P_{B}\left(\sigma_{1}\right), \quad \pi_{A}^{D p}(\sigma)=P_{A}(\sigma),
$$

where

$$
\begin{aligned}
y_{D p}^{A} & =\left(P_{D p}\right)^{A}{ }_{B} y^{B}, & \pi_{A}^{D p}=\left(P_{D p}\right)_{A}{ }^{B} \pi_{B}, \\
Q^{A} & =\left(P_{D p}\right)^{A}{ }_{B} q^{B}, & P_{A}=\left(P_{D p}\right)_{A}{ }^{B} p_{B} .
\end{aligned}
$$


Table 1: Projectors.

\begin{tabular}{cccc}
\hline Case & $\tilde{\mathcal{A}} \neq 0, \mathcal{A} \neq 0$ & $\mathcal{A}=0$ & $\tilde{\mathcal{A}}=0$ \\
\hline$\left(P_{D p}\right)_{A}{ }^{B}$ & $\delta_{A}{ }^{B}$ & $\left(\check{P}_{\mathrm{T}}\right)_{A}{ }^{B}$ & $\left(\hat{P}_{\mathrm{T}}\right)_{A}{ }^{B}$ \\
\hline
\end{tabular}

The projectors $\left(P_{D p}\right)_{A}^{B}$ are given in table 1 , while the concrete expressions for $\left(\check{P}_{\mathrm{T}}\right)_{A}^{B}$ and $\left(\hat{P}_{\mathrm{T}}\right)_{A}{ }^{B}$ are defined in equation (B.17) and (B.13), respectively.

In case (1), equation (5.3) contains all components, because $y_{D p}^{A}(\sigma)=$ $y^{A}(\sigma)$, and we introduce this notation just in order to unify expressions for all solutions.

In cases (2) and (3), corresponding to $\mathcal{A}=0$ and $\tilde{\mathcal{A}}=0$, respectively, solution (5.3) does not contain two directions determined by the vectors $\tilde{n}_{1}^{A}$ and $\tilde{n}_{2}^{A}$. These directions satisfy Dirichlet boundary conditions, while the corresponding canonically conjugated momenta are equal to zero

$$
\left.y_{1}\right|_{0} ^{\pi}=0,\left.\quad y_{2}\right|_{0} ^{\pi}=0, \quad \pi_{1}=0, \quad \pi_{2}=0
$$

where $y_{a},(a=1,2)$ is introduced in equation (4.23).

In all cases tensor

$$
\Theta^{A B}=-\frac{1}{\kappa}\left(g_{\mathrm{eff}}^{-1} B g_{D p}^{-1} P_{D p}\right)^{A B}
$$

can be written in the same form and it is manifestly antisymmetric. The corresponding metrics are given in table 2, where the particular expressions for metrics are given in Appendix A and for projectors in Appendix B.

In case (1), when both closed and open string metric, $G_{A B}$ and $G_{A B}^{\text {eff }}$, are regular, the component form of the tensor $\Theta^{A B}$ is

$$
\Theta^{A B}=\left(\begin{array}{cc}
\Theta^{i j} & \Theta^{i} \\
-\Theta^{j} & 0
\end{array}\right)
$$

Table 2: Extended metrics.

\begin{tabular}{lccc}
\hline Case & $\tilde{\mathcal{A}} \neq 0, \mathcal{A} \neq 0$ & $\mathcal{A}=0$ & $\tilde{\mathcal{A}}=0$ \\
\hline$\left(g_{\mathrm{eff}}^{-1}\right)^{A B}$ & $\left(G_{\mathrm{eff}}^{-1}\right)^{A B}$ & $\left(\check{G}_{\mathrm{eff}}^{-1} \check{P}_{\mathrm{T}}\right)^{A B}$ & $\left(G_{\mathrm{eff}}^{-1} \hat{P}_{\mathrm{T}}\right)^{A B}$ \\
$\left(g_{D p}^{-1}\right)^{A B}$ & $\left(G^{-1}\right)^{A B}$ & $\left(g^{-1}\right)^{A B}$ & $\left(G^{-1}\right)^{A B}$ \\
\hline
\end{tabular}


where

$$
\Theta^{i j}=-\frac{1}{\kappa}\left(G_{\mathrm{eff}}^{-1} \check{\Pi}_{\mathrm{T}}^{0} B G^{-1} \check{\Pi}_{\mathrm{T}}^{0}\right)^{i j}, \quad \Theta^{i}=\frac{1}{2 \kappa \tilde{\mathcal{A}}}(B \tilde{a})^{i},
$$

and

$$
\left(\check{\Pi}_{\mathrm{T}}^{0}\right)_{i}{ }^{j}=\delta_{i}{ }^{j}+\frac{a_{i} \tilde{a}^{j}}{\tilde{\mathcal{A}}} .
$$

In the basis where both ordinary and effective metrics are diagonal, (5.7) can be transformed as

$$
{ }^{\star} \Theta^{A B}=-\frac{1}{\kappa}\left({ }^{\star} G_{\text {eff }}^{-1} B^{\star} G^{-1}\right){ }^{A B}=\left(\begin{array}{cc}
\Theta^{i j} & 0 \\
0 & 0
\end{array}\right)
$$

with the same expression for $\Theta^{i j}$ as in (5.8).

The antisymmetric parameter $\Theta^{A B}$ in cases (2) and (3) has the same form in diagonal and nondiagonal representation

$$
\Theta^{A B}=\left(\begin{array}{cc}
\Theta^{i j} & 0 \\
0 & 0
\end{array}\right)
$$

where

$$
\begin{aligned}
\Theta^{i j} & =-\frac{1}{\kappa}\left(G_{\mathrm{eff}}^{-1} \Pi_{\mathrm{T}} B G^{-1} \Pi_{\mathrm{T}}\right)^{i j}, \\
\left(\Pi_{\mathrm{T}}\right)_{i}{ }^{j} & =\delta_{i}{ }^{j}-\frac{a_{i} \tilde{a}^{j}}{\tilde{a}^{2}}-\frac{4}{\tilde{a}^{2}-a^{2}}(B a)_{i}(\tilde{a} B)^{j} .
\end{aligned}
$$

The component form of the above results are in full accordance with the results of $[1,2]$.

\subsection{Noncommutativity}

From the Poisson brackets of the basic string variables (3.4), we calculate the corresponding ones of the effective variables

$$
\left\{Q^{A}(\tau, \sigma), P_{B}(\tau, \bar{\sigma})\right\}=\left(P_{D p}\right)^{A}{ }_{B} \delta_{s}(\sigma, \bar{\sigma}),
$$

where $\delta_{s}(\sigma, \bar{\sigma})=\frac{1}{2}[\delta(\sigma-\bar{\sigma})+\delta(\sigma+\bar{\sigma})]$. 
Separating the center of mass variables

$$
\left(y_{D p}^{A}\right)_{c m}=\frac{1}{\pi} \int_{0}^{\pi} d \sigma y_{D p}^{A}(\sigma), \quad y_{D p}^{A}(\sigma)=\left(y_{D p}^{A}\right)_{c m}+Y_{D p}^{A}(\sigma),
$$

we obtain

$$
\left\{Y_{D p}^{A}(\sigma), Y_{D p}^{B}(\bar{\sigma})\right\}=\Theta^{A B} \Delta(\sigma+\bar{\sigma}),
$$

where the function $\Delta(x)$ is defined as

$$
\Delta(x)= \begin{cases}-1 & \text { if } x=0 \\ 0 & \text { if } 0<x<2 \pi \\ 1 & \text { if } x=2 \pi\end{cases}
$$

From the identity

$$
\frac{\alpha}{2} \Theta^{i j} a_{j}+\Theta^{i}=0
$$

which holds for components defined in equation (5.8), we conclude that combination $F+\frac{\alpha}{2} a_{i} x^{i}={ }^{\star} F$ is a commutative variable. The same result follows directly from the diagonal form of the noncommutativity parameter (5.10).

In case (1) where extended metrics are regular there are one commutative and $p+1$ noncommutative variables. In other two cases, where either ordinary or effective extended metric are singular, the coordinates $y_{a}(a=1,2)$ satisfy Dirichlet boundary conditions and decrease the number of the $D p$ brane dimensions. Because the variable ${ }^{\star} F$ is a commutative one, there are $p-1$ noncommutative variables in the theory. The expressions for noncommutativity parameter $\Theta^{i j}$ between coordinates $x^{i}$, and $\Theta^{i}$ between $x^{i}$ and the field $F$, are unified.

\subsection{Effective theory}

Let us introduce the effective current

$$
\tilde{j}_{ \pm A}=P_{A} \pm \kappa g_{A B}^{\mathrm{eff}} Q^{\prime B}, \quad g_{A C}^{\mathrm{eff}}\left(g_{\mathrm{eff}}^{-1}\right)^{C B}=\left(P_{D p}\right)_{A}^{B},
$$

which live in subspace defined by projector $\left(P_{D p}\right)_{A}{ }^{B}$ playing the role of unity. Using solution (5.3), we correlate it with corresponding one defined 
in (3.3) and get

$$
j_{ \pm A}= \pm 2\left(\Pi_{ \pm} g_{\mathrm{eff}}^{-1}\right)_{A}{ }^{B} \tilde{j}_{ \pm B}
$$

Substituting these relations in the canonical Hamiltonian [(3.2) or (4.5)], we obtain an effective energy-momentum tensor and Hamiltonian

$$
T_{ \pm}=\mp \frac{1}{4 \kappa}\left(g_{\mathrm{eff}}^{-1}\right) A B \tilde{j}_{ \pm A} \tilde{j}_{ \pm B} \equiv \tilde{T}_{ \pm}, \quad \mathcal{H}_{\mathrm{c}}=\tilde{T}_{-}-\tilde{T}_{+} \equiv \tilde{\mathcal{H}}_{\mathrm{c}}
$$

The effective theory is defined in the phase space spanned by the coordinates $Q^{A}$ and momenta $P_{A}$. The expressions $\tilde{T}_{ \pm}$satisfy Virasoro algebra. Consequently, the effective theory is a string theory constrained to the subspace defined by projector $\left(P_{D p}\right)_{A} B$ and symmetric under $\sigma$-parity but propagating in the effective background $G_{A B} \rightarrow g_{A B}^{\mathrm{eff}}, B_{A B} \rightarrow 0$.

\subsection{Zero central charge limit $c=0(\alpha \rightarrow \infty)$}

The expressions in the non-diagonal form are technically more convenient for applying zero central charge limit $c=0(\alpha \rightarrow \infty)$. All details concerning zero central charge limit are expressed in Appendix A.2. For $c=0$ we have

$$
\begin{aligned}
\pi_{A} \rightarrow{ }^{0} \pi_{A} & =\kappa\left({ }^{0} G_{A B} \dot{y}^{B}-2 B B_{A B} y^{B}\right), \\
j_{ \pm A} \rightarrow{ }^{0} j_{ \pm A} & ={ }^{0} \pi_{A}+2 \kappa{ }^{0} \Pi_{ \pm A B} y^{\prime B},
\end{aligned}
$$

where ${ }^{0} \Pi_{ \pm A B}=B_{A B} \pm \frac{1}{2} G_{A B}$ and ${ }^{0} G_{A B}$ is defined in equation (A.18). Index ${ }^{0}$ is chosen to signify the condition $c=\beta^{\Phi}=0$.

In case (1), where both extended metrics are regular $\left(\tilde{\mathcal{A}} \rightarrow-\tilde{a}^{2} \neq 0, \mathcal{A} \rightarrow\right.$ $\left.-a^{2} \neq 0\right)$, the noncommutativity parameter takes the form

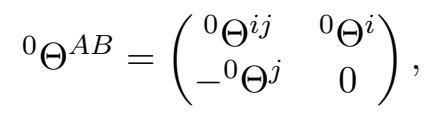

where

$$
{ }^{0} \Theta^{i j}=-\frac{1}{\kappa}\left(\tilde{P}_{\mathrm{T}} B P_{\mathrm{T}}^{0}\right)^{i j}, \quad{ }^{0} \Theta^{i}=-\frac{(B \tilde{a})^{i}}{2 \kappa \tilde{a}^{2}},
$$

and the projectors $\left(P_{\mathrm{T}}^{0}\right)_{i}{ }^{j}$ and $\tilde{P}_{\mathrm{T}}^{i j}$ are defined in equation (A.20). 
In cases (2) and (3) in the limit $c=0$ the noncommutativity parameters have the same form

$$
{ }^{0} \Theta^{A B}=\left(\begin{array}{cc}
{ }^{0} \Theta^{i j} & 0 \\
0 & 0
\end{array}\right)
$$

For case (2) of singular ${ }^{0} G_{A B}\left(\mathcal{A} \rightarrow-a^{2}=0\right)$ the noncommutativity parameter is defined as

$$
{ }^{0} \Theta^{i j}=-\frac{1}{\kappa}\left(G_{\mathrm{eff}}^{-1} P_{\mathrm{T}}^{1} B G^{-1} P_{\mathrm{T}}^{1}\right)^{i j}, \quad\left(P_{\mathrm{T}}^{1}\right)_{i}{ }^{j}=\delta_{i}{ }^{j}-\frac{4}{\tilde{a}^{2}}(B a)_{i}(\tilde{a} B)^{j},
$$

while in case (3) of singular effective extended metric ${ }^{0} G_{A B}^{\mathrm{eff}}\left(\tilde{\mathcal{A}} \rightarrow-\tilde{a}^{2}=0\right)$ we have

$$
{ }^{0} \Theta^{i j}=-\frac{1}{\kappa}\left(G_{\mathrm{eff}}^{-1} \hat{P}_{\mathrm{T}}^{1} B G^{-1} \hat{P}_{\mathrm{T}}^{1}\right)^{i j}, \quad\left(\hat{P}_{\mathrm{T}}^{1}\right)_{i}{ }^{j}=\delta_{i}{ }^{j}+\frac{4}{a^{2}}(B a)_{i}(\tilde{a} B)^{j} .
$$

Note that in the zero central charge limit identity (5.17) turns to identity $\Theta^{i j} a_{j}=0$, so that commutative variable $\frac{1}{\alpha}{ }^{\star} F=\frac{1}{\alpha} F+\frac{1}{2} a_{i} x^{i}$ turns to $a_{i} x^{i}$.

All these results are in full correspondence with the expressions obtained in framework without Liouville term [1].

\section{Concluding remarks}

In this article we considered noncommutativity properties of the space-time extended by the conformal part of the world-sheet metric $F$. The field $F$, introduced by Liouville term, allows us to establish the quantum conformal invariance without using the dilaton space-time equation of motion, $\beta^{\Phi}=0$. In fact, after imposing the space-time equation of motion, $\beta_{\mu \nu}^{G}=0$, the Liouville action cancels the remaining constant anomaly, $\beta^{\Phi}=c$, and makes the conformal part of the world sheet metric, $F$, dynamical variable.

So it is natural to consider the extended space-time with the coordinates $y^{M}=\left(x^{\mu}, F\right)$ and the metric $G_{M N}$. An extended $D p$-brane, parameterized by extended string coordinates $y^{A}=\left(x^{i}, F\right)$ with the corresponding closed string metric $G_{A B}$, is emebedded in the extended space-time. In this way in equation (5.6) we unified the expressions for noncommutativity parameter $\Theta^{i j}$ between $D p$-brane space-time coordinates $x^{i}$ with the noncommutativity parameter $\Theta^{i}$ between $x^{i}$ and the field $F$.

When the both extended metrics are regular $(\mathcal{A} \neq 0, \tilde{\mathcal{A}} \neq 0)$ the analysis in the extended formulation is completely equivalent to the dilaton free case. 
Table 3: $D p$-brane features.

\begin{tabular}{cccccc}
\hline Case & $y_{a}$ & $\left(P_{\mathrm{Dp}}\right)_{A}{ }^{B}$ & $D_{D_{p}}$ & $N_{\mathrm{nc}}$ & $y_{\mathrm{c}}$ \\
\hline 1. & - & $\delta_{A}{ }^{*}$ & $\mathrm{p}+2$ & $\mathrm{p}+1$ & ${ }^{\star} F$ \\
2. & $y_{1}, y_{2}$ & $\left(\check{P}_{\mathrm{T}}\right)_{A}{ }^{B}$ & $\mathrm{p}$ & $\mathrm{p}-1$ & ${ }^{\star} F$ \\
3. & $y_{1}, y_{2}$ & $\left(\hat{P}_{\mathrm{T}}\right)_{A}{ }^{B}$ & $\mathrm{p}$ & $\mathrm{p}-1$ & ${ }^{\star} F$ \\
\hline
\end{tabular}

Applying known results of the dilaton free case on extended $D p$-brane, we independently derived the results of $[1,2,7]$, in a much simpler way. We show that both noncommutative parameters, $\Theta^{i j}$ and $\Theta^{i}$, are just components of the extended noncommutativity parameter. One $D p$-brane coordinate, ${ }^{\star} F=F+\frac{\alpha}{2} a_{i} x^{i}$, is commutative. Consequently, number of noncommutative variables is the same as in the absence of dilaton field.

In case (2) the closed string metric $G_{A B}$ has one singular direction, whereas in case (3) there are two singular directions of the open string metric $G_{A B}^{\mathrm{eff}}$. In both cases the first class constraints appear in the theory generating local symmetries. Fixing the gauge, the first class constraints and gauge conditions behave like second class constraints. Solving the second class constraints we obtain an effective theory expressed in terms of the effective variables $Q^{A}$, symmetric under $\sigma$-parity transformation, and corresponding effective metric $g_{A B}^{\mathrm{eff}}$. We conclude that $D p$-brane is described by one commutative, ${ }^{\star} F$, and $p-1$ noncommutative coordinates.

All cases can be explained from the unique point of view. As a consequence of the relation between background fields, some coordinates $y_{a}=$ $\frac{\tilde{n}_{a A} y^{A}}{\tilde{n}_{a}^{2}}$ satisfy Dirichlet boundary conditions, and change the dimensionality of the $D p$-brane. The physical $D p$-branes are defined by projections $\left(P_{D p}\right)_{B}^{A}$ with dimensions $D_{D p}=\left(P_{D p}\right)_{A}^{A}$. The variable ${ }^{\star} F$ is commutative in all three cases, $y_{\mathrm{c}}={ }^{\star} F$, while all other directions are noncommutative, because $y_{\mathrm{Dp}}^{A}$ depends on both the coordinates $Q^{A}$ and momenta $P_{A}$ [see equation (5.3)]. So, the number of commutative coordinates $N_{\mathrm{c}}$ is equal to 1 , and the number of noncommutative coordinates is $N_{\mathrm{nc}}=D_{D p}-1$. This analysis is summarized in table 3 .

In this article we also introduced one methodological improvement and derived boundary conditions by canonical methods. Demanding that canonical Hamiltonian as time translation generator is differentiable in its canonical variables, we obtain the boundary conditions purely canonically. We treated boundary conditions as canonical constraints, so this approach seems to be more natural. The equations of motion for canonical momenta give the standard Lagrangian form of the boundary condition. 
All results of this paper agree with the corresponding ones in component notation of $[1,2,7]$. The advantage of the extended space-time approach is to write the action in the presence of dilaton field in the dilaton free form. In such a way we unify the expressions for noncommutativity parameters.

\section{Acknowledgments}

Work supported in part by the Serbian Ministry of Science and Environmental Protection, under contract No. 141036.

\section{Appendix A Extended space-time}

In this appendix we will introduce the metrics in diagonal and nondiagonal form, and the similarity transformation which connects them. Also we give the expressions for metrics in the zero central charge limit $c=0(\alpha \rightarrow \infty)$ in order to compare them with the results of [1].

\section{A.1 Extended metrics in nondiagonal and diagonal form}

Let us introduce the coordinates of the extended $D p$-brane $y^{A}$ and the corresponding background fields $G_{A B}$ and $B_{A B}$

$$
y^{A}=\left(\begin{array}{c}
x^{i} \\
F
\end{array}\right), \quad G_{A B}=\left(\begin{array}{cc}
G_{i j} & 2 a_{i} \\
2 a_{j} & \frac{4}{\alpha}
\end{array}\right), \quad B_{A B}=\left(\begin{array}{cc}
B_{i j} & 0 \\
0 & 0
\end{array}\right) .
$$

We can diagonalize $G_{A B}$ applying similarity transformation to vectors

$$
{ }^{\star} V^{A}=M^{A}{ }_{B} V^{B}, \quad{ }^{\star} V_{A}=\left[\left(M^{-1}\right)^{T}\right]_{A}{ }^{B} V_{B},
$$

where

$$
M_{B}^{A}=\left(\begin{array}{cc}
\delta^{i}{ }_{j} & 0 \\
\frac{\alpha a_{j}}{2} & 1
\end{array}\right), \quad\left(M^{-1}\right)^{A}{ }_{B}=\left(\begin{array}{cc}
\delta^{i}{ }_{j} & 0 \\
-\frac{\alpha a_{j}}{2} & 1
\end{array}\right) \quad(\operatorname{det} M=1) .
$$

From transformation laws for vectors (A.2) we can derive the corresponding ones for arbitrary tensors. Marking variables in diagonal form by $(\star)$ we 
obtain

and

$$
{ }^{\star} y^{A}=\left(\begin{array}{c}
x^{i} \\
{ }^{\star} F
\end{array}\right), \quad{ }^{\star} F=F+\frac{\alpha}{2} a_{i} x^{i},
$$

$$
{ }^{\star} G_{A B}=\left(\begin{array}{cc}
{ }^{\star} G_{i j} & 0 \\
0 & \frac{4}{\alpha}
\end{array}\right), \quad{ }^{\star} B_{A B}=B \text { BB }, \quad{ }^{\star} G_{i j}=G_{i j}-\alpha a_{i} a_{j} .
$$

The inverse of the metric $G_{A B}$ and ${ }^{\star} G_{A B}$ are of the form

$$
\left(G^{-1}\right)^{A B}=\left(\begin{array}{cc}
\left({ }^{\star} G^{-1}\right)^{i j} & -\frac{a^{i}}{2 \mathcal{A}} \\
-\frac{a^{j}}{2 \mathcal{A}} & \frac{1}{4 \mathcal{A}}
\end{array}\right), \quad\left({ }^{\star} G^{-1}\right)^{A B}=\left(\begin{array}{cc}
\left({ }^{\star} G^{-1}\right)^{i j} & 0 \\
0 & \frac{\alpha}{4}
\end{array}\right),
$$

where

$$
\left({ }^{\star} G^{-1}\right)^{i j}=G^{i j}+\frac{a^{i} a^{j}}{\mathcal{A}}, \quad \mathcal{A} \equiv \frac{1}{\alpha}-a^{2} .
$$

Because metrics $G_{A B}$ and ${ }^{\star} G_{A B}$ are connected by similarity transformation, their determinants are equal and have a form

$$
\operatorname{det} G_{A B}=\operatorname{det}^{\star} G_{A B}=4 \mathcal{A} \operatorname{det} G_{i j} .
$$

The corresponding effective metrics are

$$
\begin{gathered}
G_{A B}^{\mathrm{eff}}=G_{A B}-4\left(B G^{-1} B\right)_{A B}=\left(\begin{array}{cc}
\tilde{G}_{i j} & 2 a_{i} \\
2 a_{j} & \frac{4}{\alpha}
\end{array}\right), \\
{ }^{\star} G_{A B}^{\mathrm{eff}}={ }^{\star} G_{A B}-4\left(B^{\star} G^{-1} B\right)_{A B}=\left(\begin{array}{cc}
{ }^{\star} G_{i j}^{\mathrm{eff}} & 0 \\
0 & \frac{4}{\alpha}
\end{array}\right),
\end{gathered}
$$

where

$$
\tilde{G}_{i j}=G_{i j}^{\mathrm{eff}}-\frac{4}{\mathcal{A}}(B a)_{i}(a B)_{j}
$$

and

$$
{ }^{\star} G_{i j}^{\mathrm{eff}}=G_{i j}^{\mathrm{eff}}-\alpha a_{i} a_{j}-\frac{4}{\mathcal{A}}(B a)_{i}(a B)_{j}=\tilde{G}_{i j}-\alpha a_{i} a_{j} .
$$


The corresponding inverse ones are of the form

$$
\left(G_{\mathrm{eff}}^{-1}\right)^{A B}=\left(\begin{array}{cc}
\left(\tilde{G}^{-1}\right)^{i j}+\frac{\tilde{a}^{i} \tilde{a}^{j}}{\tilde{\mathcal{A}}} & -\frac{\tilde{a}^{i}}{2 \tilde{\mathcal{A}}} \\
-\frac{\tilde{a}^{j}}{2 \tilde{\mathcal{A}}} & \frac{1}{4 \tilde{\mathcal{A}}}
\end{array}\right), \quad\left({ }^{\star} G_{\mathrm{eff}}^{-1}\right)^{A B}=\left(\begin{array}{cc}
\left({ }^{\star} G_{\mathrm{eff}}^{-1}\right)^{i j} & 0 \\
0 & \frac{\alpha}{4}
\end{array}\right),
$$

with the space-time components

$$
\begin{aligned}
\left(\tilde{G}^{-1}\right)^{i j} & =\left(G_{\mathrm{eff}}^{-1}\right)^{i j}+\frac{4}{\tilde{\mathcal{A}}}(B \tilde{a})^{i}(\tilde{a} B)^{j}, \\
\left({ }^{\star} G_{\mathrm{eff}}^{-1}\right)^{i j} & =\left(G_{\mathrm{eff}}^{-1}\right)^{i j}+\frac{1}{\tilde{\mathcal{A}}}\left[\tilde{a}^{i} \tilde{a}^{j}+4(B \tilde{a})^{i}(\tilde{a} B)^{j}\right],
\end{aligned}
$$

and

$$
\tilde{\mathcal{A}} \equiv \frac{1}{\alpha}-\tilde{a}^{2}
$$

Because of the first relation in (A.14), we can raise the index of $a_{i}$ with both $\left(G_{\text {eff }}^{-1}\right)^{i j}$ and $\left(\tilde{G}^{-1}\right)^{i j}$

$$
\tilde{a}^{i}=\left(\tilde{G}^{-1}\right)^{i j} a_{j}=\left(G_{\mathrm{eff}}^{-1}\right)^{i j} a_{j}, \quad \tilde{a}^{2}=\tilde{a}^{i} a_{i}
$$

The determinants of the effective metrics are

$$
\operatorname{det} G_{A B}^{\mathrm{eff}}=\operatorname{det}^{\star} G_{A B}^{\mathrm{eff}}=4 \frac{\tilde{\mathcal{A}}^{2}}{\mathcal{A}} \operatorname{det} G_{i j}^{\mathrm{eff}} .
$$

\section{A.2 Extended metrics in the zero central charge limit $(c=0)$}

In the zero central charge limit all quantities (including closed and open string metric and projectors) in component form agree with the expressions in the case without Liouville term (see [1])

$$
{ }^{0} G_{A B}=\left(\begin{array}{cc}
G_{i j} & 2 a_{i} \\
2 a_{j} & 0
\end{array}\right), \quad{ }^{0} G_{A B}^{\mathrm{eff}}=\left(\begin{array}{cc}
{ }^{0} \tilde{G}_{i j} & 2 a_{i} \\
2 a_{j} & 0
\end{array}\right)
$$


while the inverse ones are

$$
\left({ }^{0} G^{-1}\right)^{A B}=\left(\begin{array}{cc}
\left(G^{-1} P_{\mathrm{T}}^{0}\right)^{i j} & \frac{a^{i}}{2 a^{2}} \\
\frac{a^{j}}{2 a^{2}} & -\frac{1}{4 a^{2}}
\end{array}\right), \quad\left({ }^{0} G_{\mathrm{eff}}^{-1}\right)^{A B}=\left(\begin{array}{cc}
\tilde{P}_{\mathrm{T}}^{i j} & \frac{\tilde{a}^{i}}{2 \tilde{a}^{2}} \\
\frac{\tilde{a}^{j}}{2 \tilde{a}^{2}} & -\frac{1}{4 \tilde{a}^{2}}
\end{array}\right),
$$

where

$$
\left(P_{\mathrm{T}}^{0}\right)_{i}{ }^{j}=\delta_{i}{ }^{j}-\frac{a_{i} a^{j}}{a^{2}}, \quad \tilde{P}_{\mathrm{T}}^{i j}=\left({ }^{0} \tilde{G}^{-1}\right)^{i j}-\frac{\tilde{a}^{i} \tilde{a}^{j}}{\tilde{a}^{2}} .
$$

The term ${ }^{0} \tilde{G}_{i j}$ is defined as

$$
{ }^{0} \tilde{G}_{i j}=G_{i j}^{\mathrm{eff}}+\frac{4}{a^{2}}(B a)_{i}(a B)_{j},
$$

while its inverse is

$$
\left({ }^{0} \tilde{G}^{-1}\right)^{i j}=\left(G_{\mathrm{eff}}^{-1}\right)^{i j}-\frac{4}{\tilde{a}^{2}}(B \tilde{a})_{i}(\tilde{a} B)_{j} .
$$

\section{Appendix B Extended space-time projectors}

In this appendix we introduce projector operators in order to separate noncommutative and nonphysical variables on the $D p$-brane as well as to express the noncommutativity parameter.

The projectors on the subspace spanned by vectors $n_{a}^{A}$, and on the orthogonal one with respect to the metric $g_{A B}$ are

$$
(\Pi)_{A}{ }^{B}=\gamma^{a b} n_{a A} n_{b}^{B}, \quad\left(\Pi_{\mathrm{T}}\right)_{A}{ }^{B}=\delta_{A}{ }^{B}-(\Pi)_{A}{ }^{B},
$$

where

$$
n_{a A}=g_{A B} n_{a}^{B}, \quad \gamma_{a b}=n_{a}^{A} g_{A B} n_{b}^{B}, \quad \gamma^{a c} \gamma_{c b}=\delta^{a}{ }_{b} .
$$

The transposed operator is

$$
\Pi_{B}^{A}=g^{A C} \Pi_{C}{ }^{D} g_{D B} .
$$




\section{B.1 Projectors on regular part of $G_{A B}$}

In both representations the extended metrics, $G_{A B}$ and ${ }^{\star} G_{A B}$, are singular for $\mathcal{A}=0$. The corresponding singular directions are

$$
n^{A}=\left(\begin{array}{c}
a^{i} \\
-\frac{\alpha a^{2}}{2}
\end{array}\right), \quad{ }^{\star} n^{A}=M_{B}^{A} n^{B}=\left(\begin{array}{c}
a^{i} \\
0
\end{array}\right)
$$

so that the vectors

$$
n_{A} \equiv G_{A B} n^{B}=\alpha \mathcal{A}\left(\begin{array}{c}
a_{i} \\
0
\end{array}\right)={ }^{\star} n_{A} \equiv{ }^{\star} G_{A B}{ }^{\star} n^{B},
$$

vanish for $\mathcal{A}=0$.

The corresponding projectors for nondiagonal and diagonal case are

$$
\left(P_{\mathrm{T}}^{0}\right)_{A}{ }^{B}=\left(\begin{array}{cc}
\delta_{i}{ }^{j}-\frac{a_{i} a^{j}}{a^{2}} & \frac{\alpha a_{i}}{2} \\
0 & 1
\end{array}\right), \quad\left({ }^{\star} P_{\mathrm{T}}^{0}\right)_{A}{ }^{B}=\left(\begin{array}{cc}
\delta_{i}{ }^{j}-\frac{a_{i} a^{j}}{a^{2}} & 0 \\
0 & 1
\end{array}\right) .
$$

The metric in the subspace orthogonal to the vector $n^{A}$ is defined as

$$
g_{A B}=\left(P_{\mathrm{T}}^{0} G\right)_{A B}, \quad\left(g^{-1}\right)^{A B}=\left(G^{-1} P_{\mathrm{T}}^{0}\right)^{A B} .
$$

The concrete expressions are

$$
g_{A B}=\left(\begin{array}{cc}
G_{i j}-\alpha \mathcal{A} \frac{a_{i} a_{j}}{a^{2}} & 2 a_{i} \\
2 a_{j} & \frac{4}{\alpha}
\end{array}\right), \quad{ }^{\star} g_{A B}=\left(\begin{array}{cc}
G_{i j}-\frac{a_{i} a_{j}}{a^{2}} & 0 \\
0 & \frac{4}{\alpha}
\end{array}\right),
$$

while the inverse ones have the same form in both representations

$$
\left(g^{-1}\right)^{A B}=\left(\begin{array}{cc}
G^{i j}-\frac{a^{i} a^{j}}{a^{2}} & 0 \\
0 & \frac{\alpha}{4}
\end{array}\right)=\left({ }^{\star} g^{-1}\right)^{A B} .
$$




\section{B.2 Projectors on regular part of $G_{A B}^{\mathrm{eff}}$}

There are two singular directions of the effective metric $G_{A B}^{\mathrm{eff}}$

$$
\tilde{n}_{1}^{A}=\left(\begin{array}{c}
2(\tilde{a} B)^{i} \\
0
\end{array}\right), \quad \tilde{n}_{2}^{A}=\left(\begin{array}{c}
\tilde{a}^{i} \\
-\frac{\alpha \tilde{a}^{2}}{2}
\end{array}\right),
$$

and of the corresponding one in diagonal representation ${ }^{\star} G_{A B}^{\mathrm{eff}}$ (A.10)

$$
{ }^{\star} \tilde{n}_{1}^{A}=\left(\begin{array}{c}
2(\tilde{a} B)^{i} \\
0
\end{array}\right), \quad{ }^{\star} \tilde{n}_{2}^{A}=\left(\begin{array}{c}
\tilde{a}^{i} \\
0
\end{array}\right) .
$$

In both representations the vectors with lower indices

$$
\tilde{n}_{1 A}=\frac{\tilde{\mathcal{A}}}{\mathcal{A}}\left(\begin{array}{c}
2(a B)_{i} \\
0
\end{array}\right)={ }^{\star} \tilde{n}_{1 A}, \quad \tilde{n}_{2 A}=\alpha \tilde{\mathcal{A}}\left(\begin{array}{c}
a_{i} \\
0
\end{array}\right)={ }^{\star} \tilde{n}_{2 A},
$$

vanish for $\tilde{\mathcal{A}}=0$.

Using the second expression (B.1) we obtain the projectors on subspace orthogonal to the vectors $\tilde{n}_{1}^{A}$ and $\tilde{n}_{2}^{A}$

$$
\left(\hat{P}_{\mathrm{T}}\right)_{A}{ }^{B}=\left(\begin{array}{cc}
\left(\hat{P}_{\mathrm{T}}\right)_{i}^{j} & \frac{\alpha a_{i}}{2} \\
0 & 1
\end{array}\right), \quad\left({ }^{\star} \hat{P}_{\mathrm{T}}\right)_{A}{ }^{B}=\left(\begin{array}{cc}
\left(\hat{P}_{\mathrm{T}}\right)_{i}{ }^{j} & 0 \\
0 & 1
\end{array}\right),
$$

where $\left(\hat{P}_{\mathrm{T}}\right)_{i}{ }^{j}=\delta_{i}{ }^{j}-\alpha a_{i} \tilde{a}^{j}-\frac{4}{\mathcal{A}}(B a)_{i}(\tilde{a} B)^{j}$.

\section{B.3 Metrics in subspace orthogonal on $\tilde{n}_{1}^{A}$ and $\tilde{n}_{2}^{A}$}

Using the definition of the extended effective metric (4.14), we obtain for non-diagonal representation

$$
\check{G}_{A B}^{\mathrm{eff}}=\left(\begin{array}{cc}
\check{G}_{i j} & 2 a_{i} \\
2 a_{j} & \frac{4}{\alpha}
\end{array}\right), \quad\left(\check{G}_{\mathrm{eff}}^{-1}\right)^{A B}=\left(\begin{array}{cc}
\left(\check{G}^{-1}\right)^{i j}+\frac{\tilde{a}^{i} \tilde{a}^{j}}{\tilde{\mathcal{A}}} & -\frac{\tilde{a}^{i}}{2 \tilde{\mathcal{A}}} \\
-\frac{\tilde{a}^{j}}{2 \tilde{\mathcal{A}}} & \frac{1}{4 \tilde{\mathcal{A}}}
\end{array}\right),
$$

where

$$
\check{G}_{i j}=G_{i j}^{\mathrm{eff}}+\frac{4}{a^{2}}(B a)_{i}(a B)_{j}, \quad\left(\check{G}^{-1}\right)^{i j}=\left(G_{\mathrm{eff}}\right)^{i j}-\frac{4}{\tilde{a}^{2}}(B \tilde{a})^{i}(\tilde{a} B)^{j} .
$$


The corresponding expressions in diagonal form are obtained by acting with similarity transformation (A.3)

$$
{ }^{\star} \check{G}_{A B}^{\mathrm{eff}}=\left(\begin{array}{cc}
\check{G}_{i j}-\alpha a_{i} a_{j} & 0 \\
0 & \frac{4}{\alpha}
\end{array}\right), \quad\left({ }^{\star} \check{G}_{\mathrm{eff}}^{-1}\right)^{A B}=\left(\begin{array}{cc}
\left(\check{G}^{-1}\right)^{i j}+\frac{\tilde{a}^{i} \tilde{a}^{j}}{\tilde{\mathcal{A}}} & 0 \\
0 & \frac{\alpha}{4}
\end{array}\right) .
$$

Applying the procedure described at the beginning of this appendix, on vectors $\tilde{n}_{1}^{A}$ and $\tilde{n}_{2}^{A}$ and on the metric $\check{G}_{A B}^{\mathrm{eff}}$, we obtain the projectors

$$
\left(\check{P}_{\mathrm{T}}\right)_{A}{ }^{B}=\left(\begin{array}{cc}
\left(P_{\mathrm{T}}\right)_{i}^{j} & \frac{\alpha a_{i}}{2} \\
0 & 1
\end{array}\right), \quad\left({ }^{\star} \check{P}_{\mathrm{T}}\right)_{A}{ }^{B}=\left(\begin{array}{cc}
\left(P_{\mathrm{T}}\right)_{i}{ }^{j} & 0 \\
0 & 1
\end{array}\right),
$$

for non-diagonal and diagonal case, respectively, where $\left(P_{\mathrm{T}}\right)_{i}{ }^{j}=\delta_{i}{ }^{j}-\frac{a_{i} \tilde{a}^{j}}{\tilde{a}^{2}}+$ $\frac{4}{\tilde{\mathcal{A}}}(B a)_{i}(\tilde{a} B)^{j}$.

\section{References}

[1] B. Nikolić and B. Sazdović, Gauge symmetries decrease the number of Dp-brane dimensions, Phys. Rev. D 74 (2006), 045024.

[2] B. Nikolić and B. Sazdović, Gauge symmetries decrease the number of Dp-brane dimensions. II. Inclusion of the Liouville term, Phys. Rev. D 75 (2007), 085011.

[3] C. G. Callan, D. Friedan, E. J. Martinec and M. J. Perry, Strings in background fields, Nucl. Phys. B 262 (1985), 593; T. Banks, D. Nemeschansky and A. Sen, Dilaton coupling and BRST quantization of bosonic strings, Nucl. Phys. B 277 (1986), 67.

[4] A. Connes, M. R. Douglas and A. Schwarz, Noncommutative geometry and matrix theory, JHEP 02 (1998), 003; M. R. Douglas and C. Hull, D-branes and the noncommutative torus, JHEP 02 (1998), 008; V. Schomerus, D-branes and deformation quantization, JHEP 06 (1999), 030; F. Ardalan, H. Arfaei and M. M. Sheikh-Jabbari, Noncommutative geometry from strings and branes, JHEP 02 (1999), 016; C. S. Chu and P. M. Ho, Non-commutative open string and D-brane, Nucl. Phys. B550 (1999), 151.

[5] F. Ardalan, H. Arfaei and M. M. Sheikh-Jabbari, Dirac quantization of open strings and noncommutativity in branes, Nucl. Phys. B576 (2000), 578; C. S. Chu and P. M. Ho, Constrained quantization of open 
string in background B field and non-commutative D-brane, Nucl. Phys. B568 (2000), 447; T. Lee, Canonical quantization of open string and noncommutative geometry, Phys. Rev. D62 (2000), 024022.

[6] N. Seiberg and E. Witten, String theory and noncommutative geometry, JHEP 09 (1999), 032.

[7] B. Sazdović, Dilaton field induces commutative Dp-brane coordinate, Eur. Phys. J. C44 (2005), 599.

[8] M. Li, Dirichlet boundary state in a linear dilaton background, Phys. Rev. D54 (1996) 1644; A. Rajaraman and M. Rozali, D-branes in linear dilaton backgrounds, JHEP 12 (1999), 005; S. Bhattacharyya, A. Kumar and S. Mahapatra, Noncommutativity in linear dilaton background, Mod. Phys. Lett. A16 (2001) 2263.

[9] E. S. Fradkin and A. A. Tseytlin, Effective field theory from quantized strings, Phys. Lett. B 158 (1985), 316; Quantum string theory effective action, Nucl. Phys. B 261 (1985), 1; M. B. Green, J. H. Scwarz, E. Witten, Superstring theory, Cambridge University Press, Cambridge, 1987; J. Polchinski, String theory, Cambridge University Press, Cambridge, 1998.

[10] B. Sazdović, Bosonic string theory in background fields by canonical methods, IJMP A20 (2005), 5501. 
